\title{
Brain Tumor Classification and Segmentation Based on Morphological Operations using Image Processing Techniques
}

\author{
Kotha Tirumala Naga Sriveni, Madala Vani Pujitha
}

\begin{abstract}
Brain Tumour is the solitary cause for the assassination of many individuals. A brain tumour is an accretion or widening of isolated cells in your brain. It can be identified by several tests like MRI (Magnetic Resonance Imaging), CT (Computer Tomography) scan along with several tests like biopsy, spinal tap etc. Classification and Segmentation activity take part a significant role in interpretation of brain tumours. In this paper the images should be taken in the form of jpeg format. The images are processed using data mining and machine learning classification methods. Previous research studies are intended as long as identifying brain tumours using dissimilar classification and segmentation approaches. The initiated system consists of certain process for recognition of the tumour. The first step is about Pre-processing and the next is about segmentation, Feature extraction is the third step in this process and Classification is used to detect the tumour. Morphological Operations are performed in this process based on the tumour size, shape and colour. Neural Network is used for classification along with -Support Vector Machine (SVM) classifiers are worn for structured recognition. Due to this we can reduce the inappropriate or false diagnosis error rate of brain tumour identification for the patients and also, we can get faster and accurate results.
\end{abstract}

Keywords: Brain Tumor, Computer Tomography, Diagnosis, Magnetic Resonance Imaging, Neural Network.

\section{INTRODUCTION}

As of late, the presentation of data innovation and e-human services framework in the clinical field encourages clinical specialists to give better social insurance to the patient. This examination tends to the issues of division of anomalous mind tissues and ordinary tissues, for example, dim issue, and cerebrospinal liquid from MR pictures utilizing highlight extraction strategy and bolster vector machine classifier. MRI is a kind of yield that usages strong appealing fields and the radio waves to the produce quick and dirty pictures in inside the body. An MRI scanner is a progressively drawn out

Revised Manuscript Received on April 25, 2020

* Correspondence Author

Kotha Tirumala Naga Sriveni*, Dept of CSE, Velagapudi Rama Krishna Siddhartha Engineering College, Vijayawada, India. Email: srivenikotha@gmail.com

Madala Vani Pujitha, Computer Science and Engineering Department,VelagapudiRamaKrishnaSiddharthaEngineeringCollege,Vijay awada,India.Email:pujitha.vani@gmail.com :

(c) The Authors. Published by Blue Eyes Intelligence Engineering and Sciences Publication (BEIESP). This is an open access article under the CC BY-NC-ND license (http://creativecommons.org/licenses/by-nc-nd/4.0/) chamber that contain a mind-boggling magnet. You lie inside the chamber amidst the range. It will when all is said in done be utilized to inspect in every way that really matters any piece of the body, including the cerebrum and spinal string, bones and joints, chests, heart and veins, inside organs, for example, the liver, paunch or prostate organ. The consequences of MRI expansiveness can be utilized to help analyse conditions, plan meds and survey how persuading past treatment has been. The tumour is basically an unregulated production of dangerous cells in every part of the body, while a tumor in the brain is an unregulated production of carcinogenic cells throughout the mind. A tumour may be good or harmful to the mind. The supportive tumour of the mind is structurally stable and contains no dynamic (disease) cells, whereas dangerous cerebral tumours are structurally non-uniform and contain dynamic cells. Gliomas and meningiomas are second-rate tumors, assigned beneficial tumors, and glioblastoma and astrocytoma are a subset of high-grade tumors considered hazardous tumours.

\section{RELATED WORK}

This section displays the state-of-art techniques and procedures found in the present design. It characterizes the most well-known methodologies. Heba Mohsen et.al [1] proposed Deep Neural Network classifier which is one of the DL structures for mentioning a dataset of 66 brain MRIs into 4 classes for example standard, glioblastoma, sarcoma and metastatic bronchogenic carcinoma tumors. The classifier was joined with the discrete wavelet Transform (DWT) the marvelous part extraction instrument and key areas assessment (PCA) and the evaluation of the execution was uncommonly amazing over all the execution measures. Doesn't contain enormous number of focus focuses and it is utilized separate average cerebrum and a few sorts of brain tumors, for example, glioblastoma, sarcoma and metastatic bronchogenic carcinoma tumors. Aaswad Sawant et.al [2] center around the utilization of tensor stream for the affirmation of psyche hazardous advancement utilizing MRI In tensor stream we understood convolution neural structure with 5 layers. Here hard and fast 1800 MRI were utilized in dataset out of which 900 were harmful and 900 were non-damaging. The availability exactness apparently was $99 \%$ and underwriting accuracy was $98.6 \%$ in 35 ages. This structure is up 'til now being made.

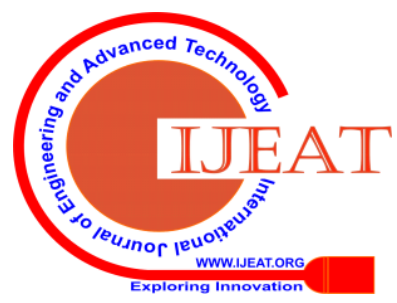


The framework can be utilized as an ensuing choice by professionals and radiologists to perceive cerebrum tumor successfully and profitably. EmanAbde-Maksoud et.al [3] presents an effective pictures division approach utilizing K-infers gathering system and joined with the Fuzzy C-infers count. It is trailed by thresholding and level set division stages to give cautious cerebrum tumor area. The proposed strategy can get focal points of the K-suggests gathering for picture division in the bits of unimportant figuring time. In like manner, it can get the advantages of the Fuzzy C-suggests in the pieces of exactness. E. Ben George eta.al [4] proposed the nature enthused count are the most remarkable for upgrade. Cuckoo Search (CS) computation is one such count which is gainful in managing progress issues in differed fields. The human psyche is the most disperse structure where seeing the tumor like illnesses are amazingly trying considering the way that segregating the sections of the cerebrum is unpredictable. The tumor may a bit of the time happen with a similar intensity of normal tissues.

M. Huang et.al [5] proposed the Brain tumor division is an essential technique for early tumor end and radiotherapy sorting out. Be that as it may, distinctive character tumor division frameworks have been appeared, improving tumor division techniques is so far testing since cerebrum tumor MRI pictures show complex attributes, for example, high traditional collection in tumor appearance and questionable tumor compels, a novel solicitation structure was accumulated by bringing the zone free projection into the standard social event model. Y. Li et.al [6] proposed the distinct division of cerebrum tumor from MR picture is sincere for the finding and treatment of brain undermining improvement. We propose a novel mechanized psyche tumor division strategy dependent on a probabilistic model joining lacking coding and Markov self-self-assured field (MRF). They plan the brain tumor division task as a pixel-wise checking issue concerning three classes: tumor, edema and solid tissue.

N. Menon et.al [7] Tumour Segmentation of MRI Brain pictures is as of not long ago an irksome issue. They proposed a snappier MRI Brain Image division philosophy dependent on Artificial Bee Colony (ABC) figuring and Fuzzy-C Means (FCM) tally. The driving force in ceaseless diminish scale between time is searched for utilizing limit estimation. Beyond what many would consider possible respect is searched for with the assistance of ABC tally. So as to get a fruitful prosperity work for $\mathrm{ABC}$ calculation the chief picture is decayed by discrete wavelet changes.

Raghavan [8] have introduced a neural system-based strategy for mind tumor location and characterization. Right now, quality rate is created independently for division of WM, GM, CSF, and tumor locale and cases an exactness of $83 \%$ utilizing neural system-based classifier. Alfonse and Salem [9] have exhibited a system for programmed characterization of mind tumor from MR pictures utilizing an SVM-based classifier. To improve the precision of the classifier, highlights are extricated utilizing quick Fourier change and decrease of highlights is performed utilizing Minimal-Redundancy-Maximal-Relevance method. Zanaty [10] proposed a procedure for cerebrum tumor division dependent on a crossover kind of approach, joining FCM, seed district developing, and Jaccard similitude coefficient calculation to quantify portioned dim issue and white issue tissues from MR pictures.

Sharma et al. [11] have displayed an exceptionally productive method which claims exactness of $100 \%$ in the order of mind tumor from MR pictures. This strategy is using surface crude highlights with counterfeit neural system (ANN) as division and classifier apparatus.

\section{METHODOLOGY}

This section shows the system of the proposed approach. Every single piece of the technique is exhibited. The proposed procedure contains 6 stages. At first Brats 2015 Data set is obtained from the official store and the pictures from the dataset are prepared with standardization. At that point the dataset is isolated into preparing and testing set. The last division map is created by foreseeing from the prepared and spared model.
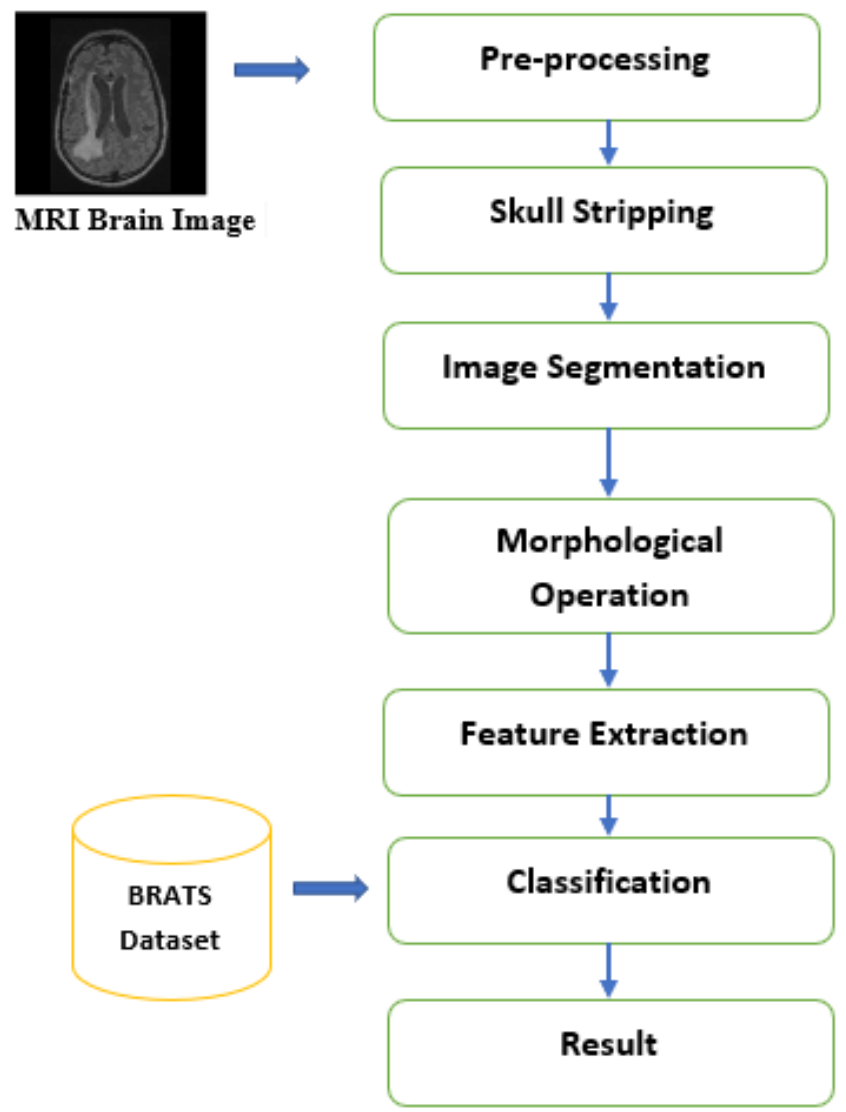

Fig 1. Structure of Proposed Methodology

Our methodology consists of seven phases they are,

- BRATS Image Data set

- Pre-processing

- Skull Peeling

- Image Segmentation

- Morphological Operations

- Feature Extraction

- Classification

\section{A. BRATS Image Data set}

At first Brats 2015 Data set is obtained from the official archive [12] and the pictures from the dataset are handled with standardization.
\& Sciences Publication

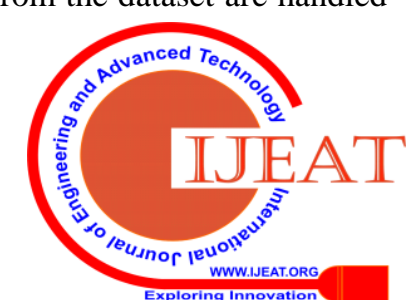


At that point the dataset is separated into preparing and testing set. BRAST 2015 setting up a set contains a total of 274 pictures in four qualities: Flair, T1, T1C and T2. Of the 274 pictures, 220 were appointed high assessment gliomas, while the remainder of the 54 was named low quality gliomas. Most of the photos have a size of $240 \times 240$.

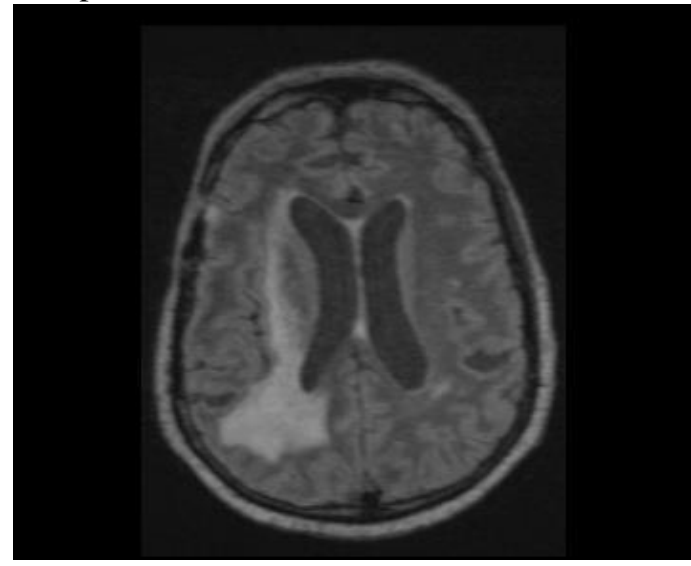

Fig 2. Sample MRI Brain Image

\section{B. Pre-processing}

The basic endeavor of Pre-taking care of is to improve the idea of the MR pictures and make it in a structure proper for extra getting ready by human or machine vision system. Moreover, Pre-getting ready helps with improving certain parameters of MR pictures, for instance, improving the sign to-fuss extent, redesigning the visual appearance of MR picture, ousting the unessential upheaval and undesired parts far out, smoothing the internal bit of the region, and ensuring its edges [5]. To improve the sign to-noise extent, and right now clearness of the unrefined MR pictures, we applied flexible separation redesign reliant on balanced sigmoid limit (ACE) from picture preparing procedures.

\section{Skull Peeling}

Skull stripping is a critical technique in biomedical picture examination, and it is required for the incredible appraisal of cerebrum tumor from the MR pictures. Skull stripping is the path toward discarding all non brain tissues in the cerebrum pictures. By skull stripping, it is possible to empty extra cerebral tissues, for instance, fat, skin, and skull in the brain pictures. There are a couple of systems available for skull stripping; a part of the notable techniques are customized skull stripping using picture structure, skull stripping reliant on division and morphological movement, and skull stripping subject to histogram examination or a breaking point regard.

\section{Segmentation and Morphological Operations}

At the present time, wavelet change is used for incredible division of cerebrum MR picture. The division of the corrupted cerebrum MR regions is practiced through the going with strides: In the underlying advance, the pre-dealt with mind MR picture is changed over into a combined picture with a breaking point for the cut-off of 128 being picked. Finally, the deteriorated region and the primary picture are both disengaged into two proportional districts and the dim pixel zone isolated from the break down movement is viewed as a psyche MR picture cloak.

\section{E. Feature Extraction}

It is the path toward social affair increasingly raised level information of an image, for instance, shape, surface, concealing, and separation. In reality, surface assessment is a huge parameter of human visual insight and AI system. It is used suitably to improve the exactness of discovering system by picking prominent features. In light of the astounding structure of improved tissues, for instance, WM, GM, and CSF in the cerebrum MR pictures, extraction of noteworthy features is an essential task. Textural disclosures and examination could improve the end, different periods of the (tumor orchestrating), and treatment response evaluation. The estimations incorporate condition for a part of the accommodating features is recorded underneath.

- Mean

- Contrast

- IDM

- Directional Moment

- Correlation

\section{F. Classification}

In this phase, we use two different classification techniques for brain tumor classification; they are CNN and SVM algorithms respectively. Here, SVM was existing algorithm for this task and CNN was our proposed algorithm for classification purpose.

\section{- Convolution Neural Network (CNN)}

In the standard neural framework, picture can't flexible. In any case, in convolution neural framework, picture can adaptable (i.e.) it will take 3D input volume to 3D yield volume (length, width, stature). The Convolution Neural Network (CNN) includes data layer, convolution layer, Rectified Linear Unit (ReLU) layer, pooling layer and totally related layer. In the convolution layer, the given data picture is secluded into various little areas. Part adroit commencement work is done in ReLU layer. Pooling layer is optional. The CNN based cerebrum tumor request is segregated into two phases, for instance, getting ready and testing stages. The amount of pictures is parceled into different class by using names name, for instance, tumor and non-tumor mind picture, etc. In the planning stage, pre-dealing with, feature exaction and request with Loss work is performed to make a desire model. Finally, the convolution neural framework is used for modified cerebrum tumor game plan. The cerebrum picture dataset is taken from BRAST enlightening record.

\section{- Support Vector Machine (SVM)}

The SVM calculation relies upon the examination of a coordinated learning system and is applied to one-class course of action issue to n-class portrayal issues. The guideline point of the SVM calculation is to change a nonlinear isolating goal into a straight change utilizing a capacity called SVM's piece work. Right now, utilized the Gaussian part work for change.

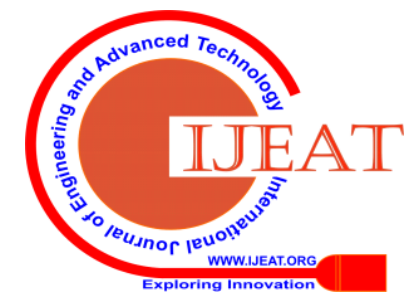




\section{Brain Tumor Classification and Segmentation Based On Morphological Operations Using Image Processing}

Techniques

By utilizing a piece work, the nonlinear examples can be changed into a high-dimensional future space where the parcel of nonlinear models or data may get possible, making the request worthwhile.

\section{RESULTS AND DISCSSION}

In this paper, our data set includes images of tumor and non-tumor MRI and is gathered from various online assets [12], such as the 2015 test data set for brain tumor Image Segmentation Benchmark (BRATS). Right now, the neural convolution system has been used to conduct order and division of brain tumors. Recreation is achieved using python language. Preparation accuracy, approval accuracy, and approval malaise are expected to conspire to discover the ability of the proposed mind tumor structure. The Support Vector Machine (SVM) based order for brain tumor discovery is implemented in the current procedure. It needs to have yield for extraction. The grouping yield is produced and precision is calculated in light of highlight confidence. The measurement time is high and the SVM dependent tumor accuracy is poor and the non-tumor identification is poor.

The Segmentation Results are as shown in below figure:
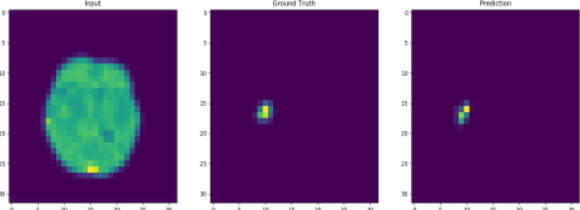

Fig 3. Output of Segmentation Image1
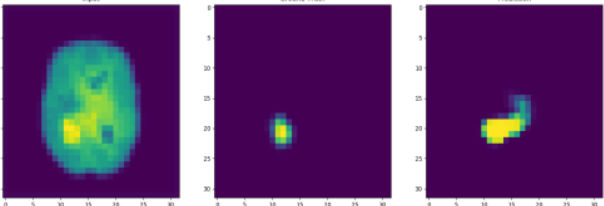

Fig 4. Output of Segmentation Image2
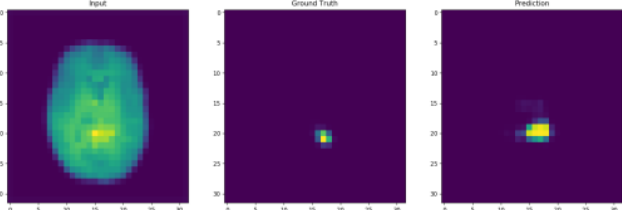

Fig 5. Output of Segmentation Image3

The Classification Results are as shown in below figure:

Published By:

Blue Eyes Intelligence Engineering

\& Sciences Publication

(C) Copyright: All rights reserved.

\begin{tabular}{|c|c|c|}
\hline Layer (type) & Output Shape & Param \# \\
\hline Conv2d_1 (Conv2D) & (None, 62, 62, 32) & 896 \\
\hline max_pooling2d_1 (MaxPooling2 & (None, 31, 31, 32) & 0 \\
\hline$\overline{C o n v 2 d \_2}$ (Conv2D) & (None, 29, 29, 32) & 9248 \\
\hline max_pooling2d_2 (MaxPooling2 & (None, 14, 14, 32) & 0 \\
\hline Conv2d_3 (Conv2D) & (None, 12, 12, 32) & 9248 \\
\hline max_pooling2d_3 (MaxPooling2 & (None, 6, 6, 32) & 0 \\
\hline flatten_1 (Flatten) & (None, 1152) & 0 \\
\hline dense_1 (Dense) & (None, 128) & 147584 \\
\hline dense_2 (Dense) & (None, 1) & 129 \\
\hline
\end{tabular}

Fig 6. Process of CNN Operation

Total params: 167,105

Trainable params: 167,105

Non-trainable params: 0

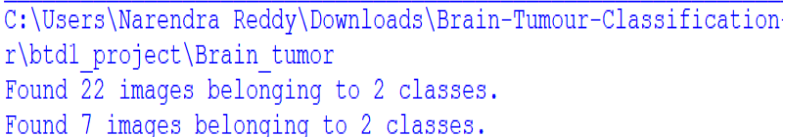

Fig 7. Parameters of CNN Operation

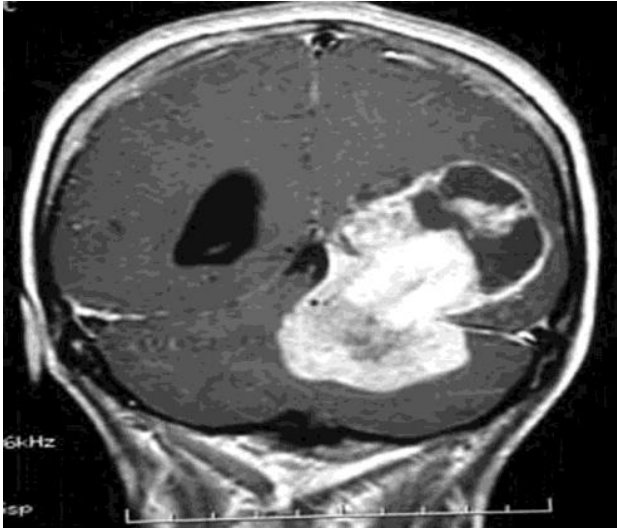

Fig 8. Brain Tumor Test Sample for Classification

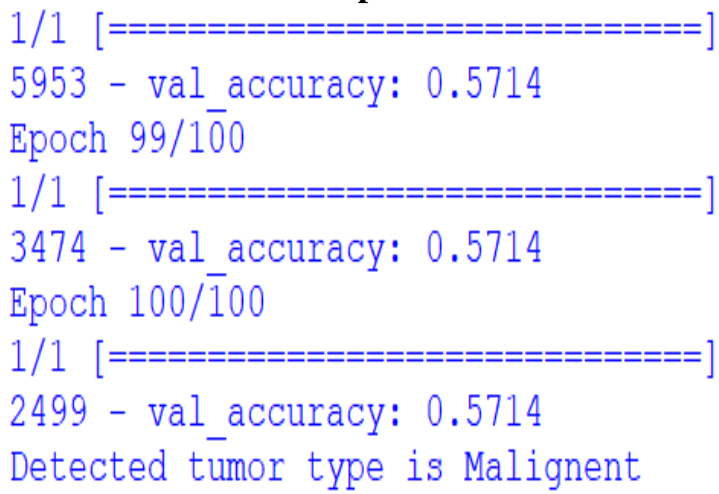

Fig 9. Classifying the tumor type is Malignant

In the proposed CNN based order doesn't require include extraction steps independently. The component esteem is taken from CNN itself. At long last, the order results as tumor cerebrum or non-tumor mind dependent on the likelihood score esteem. The typical mind picture has the most reduced likelihood score. Tumor cerebrum has most elevated likelihood score esteem, when contrasted with ordinary and tumor mind.

\section{CONCLUSION}

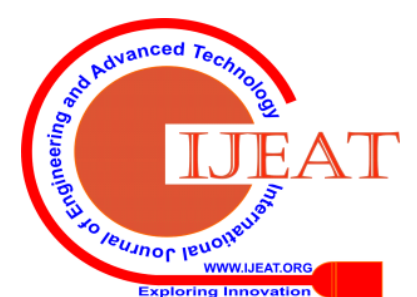


The fundamental objective of this examination work is to plan productive programmed brain tumor order with high precision, execution and low multifaceted nature. In the ordinary brain tumor arrangement is performed by utilizing Berkeley wavelet change based division, surface and highlight extraction and SVM based order are completed. The unpredictability is low. Be that as it may, the calculation time is high in the interim exactness is low. Further to improve the precision and to diminish the calculation time, a convolution neural system-based arrangement is presented in the proposed conspires. Additionally, the characterization results are given as tumor or ordinary cerebrum pictures. $\mathrm{CNN}$ is one of the profound learning strategies, which contains succession of feed forward layers. Likewise, python language is utilized for usage. BRATS database is utilized for characterization. It is one of the pre-prepared models. So, the preparation is performed for just last layer. Likewise, crude pixel esteem with profundity, width and stature include esteem are separated from CNN. At last, the Gradient average based misfortune work is applied to accomplish high exactness. The preparation precision, approval exactness and approval misfortune are determined.

\section{REFERENCES}

1. HebaMohsen, El-Sayed A. El-Dahshan, El-Sayed M. El-Horbaty, Abdel-Badeeh M. Salem," Classification using deep learning neural networks for brain tumors", Future Computing and Informatics Journal, Volume 3, Issue 1, June 2018, Pages 68-71(Science Direct).

2. AaswadSawant, MayurBhandari,RavikumarYadav, Rohan Yele, Mrs.SnehaBendale," BRAIN CANCER DETECTION FROM MRI: A MACHINE LEARNING APPROACH", International Research Journal of Engineering and Technology, Volume: 05 Issue: 04 Apr-2018.

3. Eman Abdel-Maksoud, Mohammed Elmogy, Rashid Al-Awadi, "Brain tumor segmentation based on a hybrid clustering technique", Egyptian Informatics Journal, Volume 16, Issue 1, March 2015, Pages 71-81,2015.

4. E. Ben George, G. J. Rosline and D. G. Rajesh, "Brain tumor segmentation using Cuckoo Search optimization for Magnetic Resonance Images," 2015 IEEE 8th GCC Conference \& Exhibition, Muscat, 2015, pp. 1-6, 2015.

5. M. Huang, W. Yang, Y. Wu, J. Jiang, W. Chen and Q. Feng, "Brain Tumor Segmentation Based on Local Independent Projection-Based Classification," in IEEE Transactions on Biomedical Engineering, vol. 61, no. 10, pp. 2633-2645, Oct. 2014.

6. Y. Li, Q. Dou, J. Yu, F. Jia, J. Qin and P. Heng, "Automatic Brain Tumor Segmentation from MR Images via a Multimodal Sparse Coding Based Probabilistic Model," 2015 International Workshop on Pattern Recognition in NeuroImaging, Stanford, CA, 2015, pp. 41-44, 2015.

7. N. Menon and R. Ramakrishnan, "Brain Tumor Segmentation in MRI images using unsupervised Artificial Bee Colony algorithm and FCM clustering," 2015 International Conference on Communications and Signal Processing (ICCSP), Melmaruvathur, 2015, pp. 0006-0009, 2015.

8. S. Damodharan and D. Raghavan, "Combining tissue segmentation and neural network for brain tumor detection," International Arab Journal of Information Technology, vol. 12, no. 1, pp. 42-52, 2015.

9. M. Alfonse and A.-B. M. Salem, "An automatic classification of brain tumors through MRI using support vector machine," Egyptian Computer Science Journal, vol. 40, pp. 11-21, 2016

10. E. A. Zanaty, "Determination of gray matter (GM) and white matter (WM) volume in brain magnetic resonance images (MRI)," International Journal of Computer Applications, vol. 45, pp. 16-22, 2012.

11. N. Sharma, A. Ray, S. Sharma, K. Shukla, S. Pradhan, and L. Aggarwal, "Segmentation and classification of medical images using texture-primitive features: application of BAM-type artificial neural network," Journal of Medical Physics, vol. 33, no. 3, pp. 119-126, 2008.
12. The Data set was available at https://www.smir.ch/BRATS/Start2015

\section{AUTHORS PROFILE}

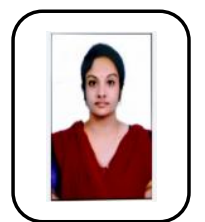

Kotha Tirumala Naga Sriveni, Pursuing M.Tech in Computer Science and Engineering at Velagapudi Rama Krishna Siddhartha Engineering College, Vijayawada. Obtained B.Tech degree in Computer Science and Engineering from NRI Institute of Technology, Pothavarappadu, Andhra Pradesh, India in 2018.

Madala Vani Pujitha has been waged as an Assistant Professor in

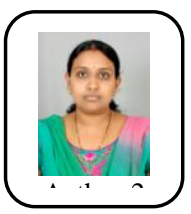
Computer Science and Engineering Department, Velagapudi Rama Krishna Siddhartha Engineering College, Vijayawada, India. She has 13 years of teaching experience and she published 8 journal papers. 